\title{
Continuous Time-of-Flight Ranging Using a MEMS Diffractive Subwavelength Period Grating (de)Modulator
}

\author{
Joris Roels, Student Member, IEEE, Ward Van der Tempel, Student Member, IEEE, \\ Daniël Van Nieuwenhove, Student Member, IEEE, Riemer Grootjans, Student Member, IEEE, \\ Maarten Kuijk, Member, IEEE, Dries van Thourhout, Member, IEEE, and Roel G. Baets, Fellow, IEEE
}

\begin{abstract}
We present a time-of-flight (TOF) ranging experiment based on optical mixing. The key component in this experiment is the optical (de)modulator. Consequently we also report on the design, fabrication, and characterization of a microelectromechanical system diffractive grating (de)modulator with a subwavelength period. The obtained results are an important step toward an optical mixing based TOF 3-D imaging system, which we will further develop in our future work.
\end{abstract}

Index Terms-Microelectromechanical devices, optical modulation, 3-D imaging, time-of-flight (TOF).

\section{INTRODUCTION}

C ONTINUOUS time-of-flight (TOF) range finding systems for 3-D imaging rely on measuring the phase shift of a modulated carrier wave induced by traveling time [1]. Essentially the 3-D imaging sensor consists of two components: a light source and a detector. The emitted light is modulated and travels toward the scene where it is reflected and travels back toward a detector. At the detector side a homodyne phase delay detection is performed, thus retrieving information on the traveling time and hence providing 3-D imaging ability.

This homodyne phase shift detection scheme requires mixing of the received (reflected) signal with the initial modulation frequency of the emitted light signal, so by consequence the mixer/(de)modulator acts as the key component in this detection scheme. Two main research tracks can be identified: modulators in the electrical domain [2], [3] and modulators in the optical domain [4]. The former is the standard approach in commercially available 3-D imaging sensors [5]; the latter allows combining off-the-shelf high-performance image sensors with a separate

Manuscript received June 25, 2008. Current version published October 31, 2008. This work was supported by the Research Foundation Flanders (FWOVlaanderen).

J. Roels, D. van Thourhout, and R. G. Baets are with the Department of Information Technology, Ghent University-IMEC, B-9000 Gent, Belgium (e-mail:_joris.roels@intec.ugent.be; dries.vanthourhout@intec.ugent.be; roel.baets@intec.ugent.be).

W. Van der Tempel, D. Van Nieuwenhove, R. Grootjans, and M. Kuijk are with Vrije Universiteit Brussel, Department of Electronics and Informatics (ETRO), Laboratory for Micro- and Opto-electronics (LAMI), BE-1050 Brussels, Belgium (e-mail: wvdtemp@etro.vub.ac.be; davnieuw@etro.vub.ac.be; rgrootja@etro.vub.ac.be; mkuijk@vub.ac.be).

Color versions of one or more of the figures in this letter are available online at http://ieeexplore.ieee.org.

Digital Object Identifier 10.1109/LPT.2008.2004348 optical modulator, ultimately providing very high image resolution and system performance compared to the electrical mixing approach.

In this letter, we focus on the second approach and present a microelectromechanical system (MEMS) optical diffractive grating modulator with a grating period smaller than the wavelength used. With this approach, we obtained sufficient modulation depth $(35 \%)$ and modulation frequency $(3.73 \mathrm{MHz})$. Moreover, we applied this component in a TOF experiment and delivered a proof-of-principle for TOF ranging with optical mixing by characterizing fibers with different lengths successfully. To our knowledge, this is the first successful demonstration of TOF with optical mixing based on MEMS.

\section{OPTICAL MEMS (DE)MODULATOR}

\section{A. MEMS Diffractive Grating Modulator Principle}

As shown in Fig. 1, the device basically consists of a diffractive grating, which is underetched over a certain region (freestanding section). The top layer in which the grating is defined is electrically isolated from the substrate by a buried oxide layer (and by air in the freestanding section). The diffractive grating itself is formed by two intersecting combs that are also electrically isolated from each other and have separate metal contacts.

By applying a voltage between such a contact and the substrate, a closing gap capacitor is formed and the corresponding beams are pulled toward the substrate. This way we create an alternating pattern of deflected and not-deflected beams, such that the incident light experiences a change in reflection. Since the grating pitch is smaller than the wavelength, only one diffraction order is present in contrast to the classical grating light valve [6].

\section{B. Modulator Design}

Application of both a DC and AC voltage (frequency $f$ ) will cause a sinusoidal force with magnitude $F_{\omega}=C V_{\mathrm{AC}} V_{\mathrm{DC}} / h$ (driving capacitance $C=\varepsilon_{0} \mathrm{lw} / \mathrm{h}, \varepsilon_{0}$ vacuum permittivity), and hence the diffractive grating acts as a modulator $\left(V_{\mathrm{AC}}=8 \mathrm{~V}\right.$, $V_{\mathrm{DC}}=300 \mathrm{~V}, l=22 \mu \mathrm{m}, h=2 \mu \mathrm{m}$ ). Using an analytical spring-mass-damper model [7] and the above-mentioned parameters, we find a theoretical resonance frequency of $3.4 \mathrm{MHz}$ and a typical vibration amplitude of $240 \mathrm{~nm}$ (Young's modulus for monocrystalline silicon in $<100\rangle$ direction $=130 \mathrm{GPa}$, mass density $=2400 \mathrm{~kg} / \mathrm{m}^{3}$ ). Taking into account this deflection and using a rigorous coupled-wave analysis software tool, 


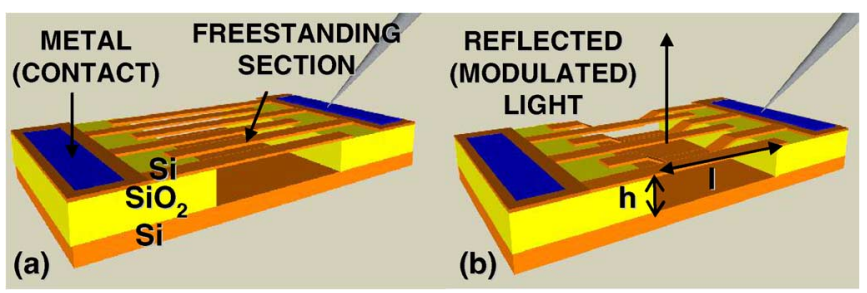

Fig. 1 MEMS diffractive grating modulator principle: (a) not-actuated versus (b) actuated state.

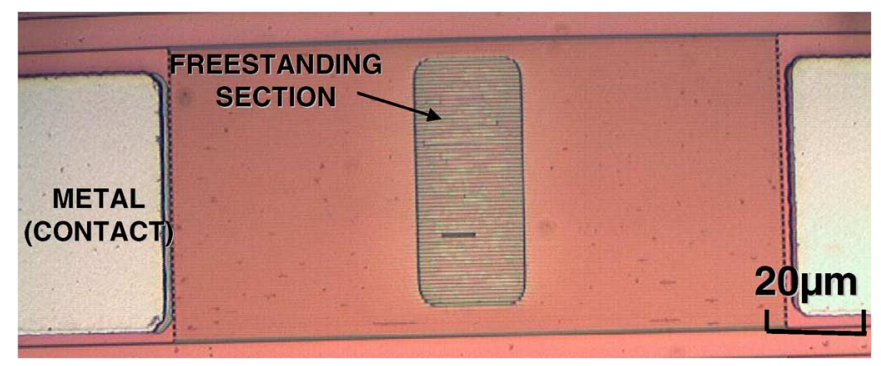

Fig. 2. Top view of fabricated diffractive grating (de)modulator.

we calculated the reflections for both the not-actuated and actuated state and found modulation depths of $57 \%$ (transverse electric (TE), E-field parallel to grating beams) and $31 \%$ (transverse magnetic).

\section{Modulator Fabrication}

In a silicon-on-insulator wafer (top layer monocrystalline $\mathrm{Si}$ : $t=220 \mathrm{~nm}+$ buried oxide layer: thickness $h=2 \mu \mathrm{m}) \mathrm{a}$ diffraction grating with 750-nm period and fill-factor $66 \%$ (so beam width $w=500 \mathrm{~nm}$ ) is defined using a deep-ultraviolet lithography process in a standard research complimentary metal-oxide-semiconductor fab [8]. Next the metal contacts are defined (stack of 150-nm Al $+100 \mathrm{~nm} \mathrm{Au}$, both evaporated) using a standard lithography and lift-off process. To define the freestanding regions (+-22 $\mu \mathrm{m}$ string length 1 ), we applied a resist mask after a treatment in a vacuum oven with gaseous hexamethyldisilizane (= adhesion promoter) to ensure good adhesion of the mask. The underetch is performed with wet buffered $\mathrm{HF}$ and the samples are dried afterwards using a $\mathrm{CO}_{2}$ critical-point-drying process to prevent stiction and damage due to surface tension. A microscope picture of such a processed device is shown in Fig. 2.

\section{Modulator Characterization}

Continuous-wave (CW) light (wavelength $\lambda=1542 \mathrm{~nm}, \mathrm{TE}$ ) was focused with a lensed fiber onto the grating modulator. With a probe needle an AC-voltage was applied such that the grating reflection varies in time as $R(t)=R_{\mathrm{CW}}+R_{\text {mod }} \sin (2 \pi f t)$. The reflected light was collected with the same fiber and separated from the incoming light using a circulator, so for the reflected signal we find $(\alpha=$ attenuation factor, $P_{\text {init }}=$ the initial laser power)

$$
P_{\mathrm{OUT}}=P_{\mathrm{init}} \alpha\left[R_{\mathrm{CW}}+R_{\mathrm{mod}} \sin (2 \pi f t)\right] .
$$

Using an optical detector + electrical spectrum analyzer, the optical CW power $P_{\mathrm{CW}}\left(=P_{\text {init }} \alpha R_{\mathrm{CW}}\right)$ was recorded in parallel with the modulated optical power $P_{\text {mod }},\left(=P_{\text {init }} \alpha R_{\text {mod }}\right)$ so finally the modulation depth $P_{\mathrm{mod}} / P_{\mathrm{CW}}=R_{\mathrm{mod}} / R_{\mathrm{CW}}$, could be determined in function of the frequency of the AC-voltage while the voltage amplitudes were kept constant at $V_{\mathrm{AC}}=8 \mathrm{~V}$ and $V_{\mathrm{DC}}=300 \mathrm{~V}$. Sweeping the modulation frequency we attained a maximal modulation depth of $35 \%$ at $3.73 \mathrm{MHz}$ which corresponds quite well to the previously calculated theoretical natural beam frequency $(3.4 \mathrm{MHz})$. The deviation between theory and experiment for the modulation depth might be explained by the fact that the curvature of the beams was ignored in the optical simulation.

\section{TIME-OF-FLIGHT}

\section{A. TOF Principle}

In principle, the experimental setup for our TOF experiment is very similar to the setup used for the characterization of the modulator, however, the light source itself is now modulated and reaches the mixer with a phase delay $\varphi(L)$ dependent on the fiber length $L$ (with $c$ the speed of light in vacuum and $n \approx 1.45$ the mode index of the optical fiber)

$$
\begin{aligned}
P_{\text {init }} & =P_{\mathrm{AVG}}+P_{\mathrm{AMP}} \sin (2 \pi f t-\varphi[L]) \\
\varphi[L] & =2 \pi f n L / c .
\end{aligned}
$$

Now we apply a phase difference $\psi$ between modulated laser source and mixer such that $R(t)=R_{\mathrm{CW}}+R_{\bmod } \sin (2 \pi f t+\psi)$ and substitute the modified expression for $R(t)$ together with (2) into (1). Applying some elementary goniometric calculus and grouping all time-dependent terms in a rest term $O(t)$ gives the following result for the demodulated light:

$$
P_{\mathrm{OUT}}=\alpha R_{\mathrm{CW}} P_{\mathrm{AVG}}+\frac{1}{2} \alpha R_{\mathrm{mod}} P_{\mathrm{AMP}} \cos (\varphi+\psi)+O(t) .
$$

Since the output power has a CW component $\sim \cos (\varphi+\psi)$, we can calculate $\varphi$ by measuring the $\mathrm{CW}$ power in $P_{\mathrm{OUT}}$ for alternating $\psi\left(0^{\circ}, 90^{\circ}, 180^{\circ}, 270^{\circ}\right)$ with a power detector and substituting the values in the following formula:

$$
\varphi=\arctan \left[\frac{\left(P_{270^{\circ}}-P_{90^{\circ}}\right)}{\left(P_{0^{\circ}}-P_{180^{\circ}}\right)}\right] .
$$

This procedure is known as the four-bucket algorithm [9]. Using (3), it is possible to extract the fiber length $L$.

\section{B. Choice of Modulation Frequency/Modulator Technology}

A maximum nonambiguous distance $L_{\mathrm{NA}}$ is determined by replacing $\varphi$ with $2 \pi$ in (3) for phase can only be measured unambiguous over a range of $2 \pi$. For a modulation frequency $f=3.73 \mathrm{MHz}$, we find $L_{\mathrm{NA}}=c /(n f) \approx 55 \mathrm{~m}$. Since $L_{\mathrm{NA}}$ increases with lower $f$, one might think that $f$ should be chosen as low as possible; however, this is not the case because a larger 


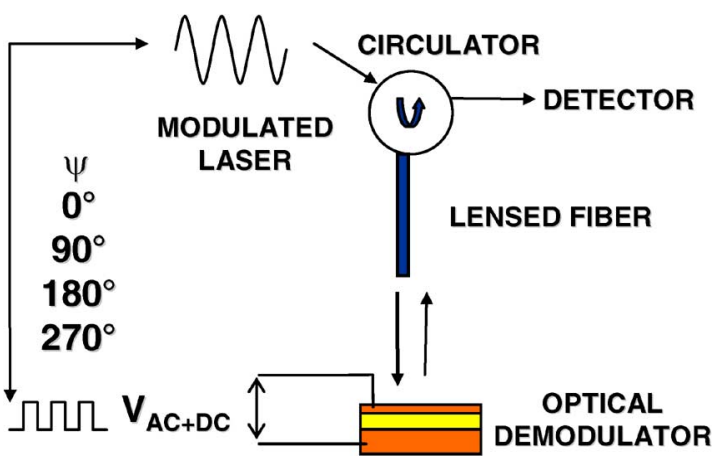

Fig. 3 TOF experimental setup.

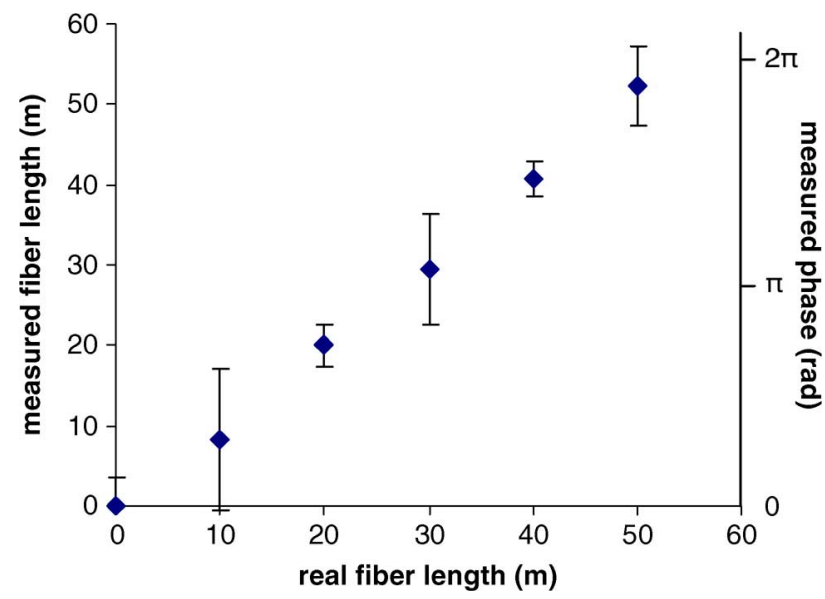

Fig. 4 TOF experimental results: real fiber length versus measured phase (right axis) and extracted fiber length (left axis).

$L_{\mathrm{NA}}$ would result in a lower depth resolution. A typical compromise value for $f$ lies in the megahertz range, so MEMS are a good choice for implementing the optical modulator.

\section{Experimental Results}

The setup is shown in Fig 3. The light of the modulated laser source $(\lambda=1542 \mathrm{~nm}, \pm 2 \mathrm{~mW})$ is focused with a lensed fiber onto the grating modulator and the $\mathrm{CW}$ component of the demodulated light is measured for different $\psi$.

Finally, from our measurement data, we calculated the fiber length and compared with the real (known) value. The experiment was repeated for fibers of different lengths $(10,20,30,40$, $50 \mathrm{~m}$ ). The results are summarized in Fig. 4.

\section{DISCUSSION}

From Fig 4, we see that we found a good match between the experimentally extracted fiber lengths and the real fiber lengths. For example, for the 40-m fiber, the relative error between the real value and the average measured value is only $0.6 \%$. Given the fact that there is also uncertainty on the exact mode index of the fiber (estimated at $n \approx 1.45$ ), this is a good result and a significant improvement compared to our previous work [10].

The critical point to achieve low standard deviation (error bars) on the measurement data is to reduce the random (not related to TOF) fluctuations in the $\mathrm{CW}$ power level. For this reason, the lensed fiber needs to be antireflection-coated because otherwise the cavity that is formed between the lensed fiber and the grating causes fluctuations in the power level.

Furthermore, it is interesting to note that the standard deviation tends to be significantly bigger for the fibers with lengths 10,30 , and $50 \mathrm{~m}$ compared to the fibers with lengths 20 and $40 \mathrm{~m}$ (e.g., $\sigma_{30 \mathrm{~m}}=6.9 \mathrm{~m}$ versus $\sigma_{40 \mathrm{~m}}=2.1 \mathrm{~m}$ ). In the case of 20 and $40 \mathrm{~m}$, only one fiber was involved while the other lengths where achieved using a concatenation of multiple fibers. Hence we suspect that the reflections at the connectors cause fluctuations in the $\mathrm{CW}$ power level due to cavity effects.

Since we only need to detect a $\mathrm{CW}$ power, it is possible to replace the optical power detector in this scheme with a camera. In this case, each pixel allows extraction of the light traveling distance and hence a powerful 3-D imaging ability. In order to process a complete scene, the fiber system should be replaced with a free space lens system and beam splitter. Hence the reflection-related noise might decrease; however, the optical power budget will now depend on the source and system étendue and the lens system collection efficiency.

\section{CONCLUSION}

We have demonstrated a TOF ranging system with optical mixing using a MEMS diffractive grating (de)modulator. In our future work, we will work toward a free space demodulating system, which will (in combination with an off-the-shelf image sensor) result in a 3-D imaging sensor.

\section{REFERENCES}

[1] B. Hostica, P. Seitz, and A. Simoni, Optical Time-of-Flight Sensors for Solid-State 3D-vision, C. A. Grimes, E. C. Dickey, and M. V. Pishko, Eds. Stevenson Ranch, CA: American Scientific Pub., 2005, vol. 7, Encyclopedia of Sensors, pp. 259-289.

[2] R. Schwarte, "Dynamic 3D-vision," in Proc. Int. Symp. Electronic Devices for Microwave and Optoelectronics Applications, Vienna, Austria, 2001, pp. 241-248.

[3] D. Van Nieuwenhove, W. van der Tempel, R. Grootjans, J. Stiens, and M. Kuijk, "Photonic demodulator with sensitivity control," IEEE Sensors, vol. 7, no. 3, pp. 317-318, Mar. 2007.

[4] R. Gvili, A. Kaplan, E. Ofek, and G. Yahav, "Depth keying," Proc. SPIE, vol. 5006, pp. 564-574, 2003.

[5] T. Oggier, M. Lehmann, R. Kaufmann, M. Schweizer, M. Richter, P. Metzler, G. Lang, F. Lustenberger, and N. Blanc, "An all-solid-state optical range camera for 3D real-time imaging with sub-centimeter depth resolution (SwissRangerTM)," Proc. SPIE, vol. 5249, no. 65, pp. 534-545, 2004

[6] O. Solgaard, F. S. A. Sandejas, and D. M. Bloom, "Deformable grating modulator," Opt. Lett., vol. 17, no. 9, pp. 688-690, 1992.

[7] J. M. Gere and S. P. Timoshenko, Mechanics of Materials, 4th ed. Boston, MA: PWS, 1997.

[8] W. Bogaerts, R. Baets, P. Dumon, V. Wiaux, J. Beckx, D. Taillaert, B. Luyssaert, J. Van Campenhout, P. Bienstman, and D. Van Thourhout, "Nanophotonic waveguides in silicon-on-insulator fabricated with CMOS technology," J. Lightw. Technol., vol. 23, no. 1, pp. 401-412, Jan. 2005.

[9] K. Creath, Phase-Measurement Interferometry Techniques, E. Wolf, Ed. Amsterdam, The Netherlands: Elsevier, 1988, vol. XXVI, Progress in Optics.

[10] J. Roels, W. Van der Tempel, D. Van Nieuwenhove, R. Grootjans, M. Kuijk, D. van Thourhout, and R. Baets, "A Dynamic subwavelength pitch grating modulator for continuous time-of-flight ranging with optical mixing," in Proc. IEEE/LEOS Optical MEMS and Nanophotonics 2008 Conf., Freiburg, Germany, 2008. 